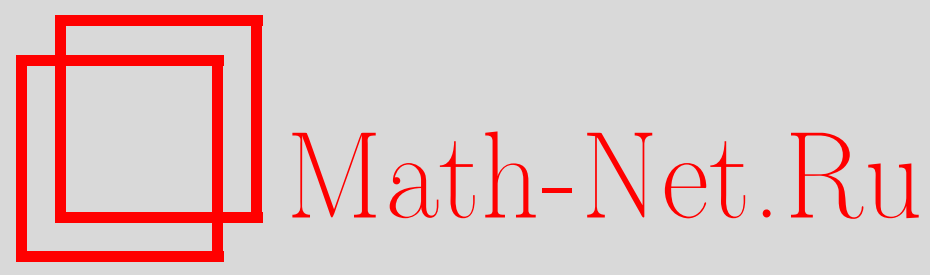

Н. Темиргалиев, Об эффективности алгоритмов численного интегрирования, связанных с теорией дивизоров в круговых полях, Матем. заметки, 1997, том 61, выпуск 2, 297301

DOI: https://doi.org/10.4213/mzm1502

Использование Общероссийского математического портала Math-Net.Ru подразумевает, что вы прочитали и согласны с пользовательским соглашением http://www . mathnet.ru/rus/agreement

Параметры загрузки:

IP : 54.224 .187 .69

26 апреля 2023 г., 16:11:12

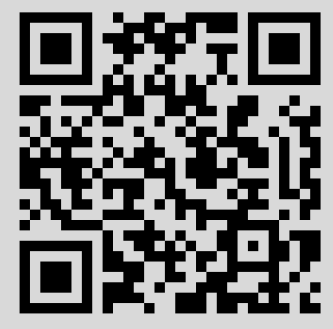




\section{ОБ ЭФФЕКТИВНОСТИ АЛГОРИТМОВ ЧИСЛЕННОГО ИНТЕГРИРОВАНИЯ, СВЯЗАННЫХ С ТЕОРИЕЙ ДИВИЗОРОВ В КРУГОВЫХ ПОЛЯХ \\ Н. Темиргалиев}

Для классов функций с доминирующей смешанной производной и с ограниченной смешанной разностью в метрике $L^{q}(1<q \leqslant 2)$ построены такие квадратурные формулы, чтобы одновременно обеспечивалась простота сетки, эффективность и близость к оптимальнму алгоритму построения сетки и неулучшаемость порядка погрешности в степенной шкале. Ранее был изучен случай $q=2$.

Библиографоия: 16 названий.

В данной работе получены квадратурные формулы для классов периодических функций $s(s \leqslant 18)$ переменных: $W_{q, s}^{r}-$ с доминирующей смешанной производной и $H_{q, s}^{r}-\mathrm{c}$ ограниченной смешанной разностью в метрике $L^{q}(1<q \leqslant 2)$.

Класс $W_{q, s}^{r}(r>0,1 \leqslant q \leqslant \infty)$ есть множество всех функций $f(x)$, представимых в виде свертки

$$
f(x)=\int_{[0,1]^{s}} \varphi(x+t)\left[2^{s} \sum_{k \in \mathbb{Z}_{+}^{s}} \prod_{j=1}^{s}\left(2 \pi k_{j}\right)^{-r} \cos 2 \pi\left(k_{j} t_{j}-\frac{r}{4}\right)\right] d t,
$$

где

$$
\|\varphi\|_{L^{q}(0,1)^{s}} \leqslant 1, \quad \mathbb{Z}_{+}^{s} \equiv\left\{\left(k_{1}, \ldots, k_{s}\right): k_{j} \in \mathbb{Z}, k_{j}>0(j=1, \ldots, s)\right\} .
$$

Класс $H_{q, s}^{r}(r>0,1 \leqslant q \leqslant \infty)$ есть множество всех 1-периодических по каждой переменной функций $f(x) \in L^{q}(0,1)$ таких, что для некоторого $l>r$ вьполнено соотношение

$$
\left\|\Delta_{h}^{l} f(x)\right\|_{L^{q}(0,1)^{s}} \leqslant \prod_{j=1}^{s}\left|h_{j}\right|^{r}
$$

где $h=\left(h_{1}, \ldots, h_{s}\right), \Delta_{h}^{l} f(x)$ - смешанная $l$-я разность с шагом $h_{j}$ по переменной $x_{j}$.

Как обычно, через $c(\cdot)$ будем обозначать положительные величины, зависящие лишь от указанньх в скобках параметров, различные, вообще говоря, в разных случаях употребления. Запись $A \ll B, B \geqslant 0$ означает $|A| \leqslant c(\alpha, \beta, \ldots) B$, а $A \ll B \ll A$ эквивалентно $A \asymp B$.

$\mathrm{K}$ настоящему времени для классов $W_{q, s}^{r}(2 \leqslant q<\infty, r>0)$ и $H_{q, s}^{r}(1<q \leqslant \infty$, $r>1)$ найдены правильные порядки убывания погрешностей оптимальных квадратурных формул (см., например, [1]-[5]). 
Цель работы состоит в построении таких квадратурных формул, чтобы одновременно обеспечивалась простота сетки, эффективность и близость к оптимальному алгоритма построения сетки и неулучшаемость порядка погрешности в степенной шкале.

Искомые сетки определяются равенством $(\{\cdot\}$ - дробная часть)

$$
\left(\left\{\frac{n}{p} a_{1}\right\}, \ldots,\left\{\frac{n}{p} a_{s}\right\}\right) \quad(n=1, \ldots, p)
$$

где $a_{1}, \ldots, a_{s}$ - специальным образом выбранные целые числа (оптимальные коэффициенты по модулю $p$ ).

Сетки (1) были введены Н. М. Коробовьп [6]. Применению этих сеток в различных задачах анализа посвящена литература (см., например, [1]-[14] и содержащуюся в них библиографию).

Тем не мене, в обзоре [12] 1988 года Вань Юань указал: “По-видимому, центральной проблемой в численном интегрировании является нахождение прямых методов для получения оптимальных коэффициентов".

Эффективный алгоритм для вычисления оптимальных коэффициентов относительно произвольного положительного числа $T \geqslant c(l)$ за $T \ln \ln T$ элементарных арифметических операций такой, что $p \leqslant T$, представлен в [14] и заключается в следующем. Ниже используются определения алгебраической теории чисел и обозначения из [14], в частности, $l=s+1 \leqslant 19-$ простое число,

$$
\begin{gathered}
\theta=\cos \frac{2 \pi}{l}+i \sin \frac{2 \pi}{l}, \omega_{\nu}=\theta^{\nu-1} \quad(\nu=1, \ldots, s), \\
N(m)=N\left(m_{1}, \ldots, m_{s}\right)=\prod_{k=1}^{s}\left(m_{1}+\theta^{k} m_{2}+\cdots+\theta^{k(s-1)} m_{s}\right)
\end{gathered}
$$

- норма главного идеала $(m)$ - положительное число при $m \in \mathbb{Z}^{s}, m \neq 0$.

1. Находится наименьшее простое $p, p \equiv 1(\bmod l)$ такое, что $p$ не делит произведение $\prod N(m)$, где $m$ пробегает всевозможные $m \in \mathbb{Z}^{s}, m \neq 0, \prod_{j=1}^{s} \max \left(1 ;\left|m_{j}\right|\right) \leqslant R$, $c(s) R \ln ^{s} R \leqslant T$.

2. Перебором по целым $m_{j},\left|m_{j}\right| \leqslant c p^{1 / s}(j=1, \ldots, s)$, находится такое $n=n_{1} \omega_{1}+\cdots$ $+n_{s} \omega_{s}$, что для главного идеала $(n)$ вьполнено $N(n)=p$.

3. Из соотношений $(k=1, \ldots, s)$

$$
\gamma_{k}=n \omega_{k}=c_{k 1}+c_{k 2} \theta+\cdots+c_{k s} \theta^{s-1}
$$

выписьвается матрица

$$
d^{\prime}=\left(\begin{array}{ccc}
c_{11} & \ldots & c_{s 1} \\
\vdots & \ddots & \vdots \\
c_{1 s} & \ldots & c_{s s}
\end{array}\right)
$$

4. Для решетки с базисом $\gamma_{j}^{\prime}=\sum_{k=1}^{s} c_{k j} \omega_{k}(j=1, \ldots, s)$ выписьвается базис

$$
\gamma_{k}^{\prime \prime}=\sum_{j=1}^{s} v_{k j} \omega_{j} \quad(k=1, \ldots, s)
$$


с целочисленной треугольной матрищей $\left\{v_{k j}\right\}_{k, j=1}^{s}$, затем из равенства $p=\left|v_{11} \ldots v_{s s}\right|$ находится номер $j_{0}$, что $\left|v_{j_{0} j_{0}}\right|=p$.

5. Находятся коэффициенты

$$
a_{k}=(-1)^{k+j_{0}} M_{j_{0} k} \operatorname{sign} \operatorname{det} d^{\prime} \quad(k=1, \ldots, s),
$$

где $M_{j k}$ есть дополнительный минор элемента $c_{j k}$ матрицы $d^{\prime}$.

На основе этого алгоритма доказана следующая теорема.

ТЕорема. Пусть $l=s+1 \leqslant 19$ - простое число, $1<q \leqslant 2, r q>1$. Тогда для всякого $T \geqslant c(l)$ простое $p, p \equiv 1(\bmod l), p \leqslant T$, и иелье числа $a_{1}, \ldots, a_{s}$, отыскиваемые согласно алгоритму $1-5$ за $T \ln \ln T$ әлементарных арифметических операчий, таковы, что выполняются следующие неравенства:

$$
\sup _{x(t) \in W_{q, s}^{r}}\left|\int_{[0,1]^{s}} x(t) d t-\frac{1}{p} \sum_{n=1}^{p} x\left(\left\{\frac{n}{p} a_{1}\right\}, \ldots,\left\{\frac{n}{p} a_{s}\right\}\right)\right| \ll \frac{(\ln T)^{s r+(s-1) / q}}{T^{r}}
$$

$u$

$$
\sup _{x(t) \in H_{q, s}^{r}}\left|\int_{[0,1]^{s}} x(t) d t-\frac{1}{p} \sum_{n=1}^{p} x\left(\left\{\frac{n}{p} a_{1}\right\}, \ldots,\left\{\frac{n}{p} a_{s}\right\}\right)\right| \ll \frac{(\ln T)^{s r+(s-1)}}{T^{r}}
$$

ДокАЗАТЕЛЬСТВо. Эта теорема при $q=2$ доказана в $[14$, теоремы 6,7$]$. При $1<q<2$ дополнительно применяются неравенство Хаусдорфа-Юнга для рядов Фурье [15, с. 172]. Поэтому будем пользоваться обозначениями, леммами и теоремами из [14] (в приведенной там нумерации).

Сначала докажем первое утверждение.

Выберем $R=2^{\theta}$ таким, что вьполнено равенство $T=c(s) R \ln ^{s} R$. Тогда в силу теорем 3,2 и 4 применением алгоритма $1-5$ за $\leqslant c(s) T \ln \ln T$ элементарных арифметических операций находятся простое $p \leqslant T$ и целые $a_{1}, \ldots, a_{s}$ такие, что

$$
\begin{aligned}
\Delta & \equiv\left|\int_{[0,1]^{s}} x(t) d t-\frac{1}{p} \sum_{n=1}^{p} x\left(\left\{\frac{n}{p} a_{1}\right\}, \ldots,\left\{\frac{n}{p} a_{s}\right\}\right)\right| \\
& \ll \sum_{\tau_{1}+\cdots+\tau_{s} \geqslant \theta} \sum_{m \in \rho(\tau)}|\widehat{x}(m)| \chi_{\mathfrak{A}}(m) .
\end{aligned}
$$

Далее, применяя неравенство Гёльдера, неравенство Хаусдорфа-Юнга, а также неравенство (см. [16, с. 93])

$$
\left\|\sum_{\tau: \tau_{1}+\cdots+\tau_{s}=k} \sum_{m \in \rho(\tau)} \widehat{x}(m) e^{2 \pi i(m, t)}\right\|_{L^{q}(0,1)^{s}} \ll 2^{-k r},
$$

справедливое для всякой функции $x(t) \in W_{q, s}^{r}(1<q<\infty)$, получим

$$
\begin{aligned}
\Delta & \leqslant \sum_{k \geqslant \theta}\left\|\sum_{\tau_{1}+\cdots+\tau_{s}=k} \sum_{m \in \rho(\tau)} \widehat{x}(m) e^{2 \pi i(m, t)}\right\|_{L^{q}(0,1)^{s}}\left(\sum_{\tau_{1}+\cdots+\tau_{s}=k} \sum_{m \in \rho(\tau)} \chi_{\mathfrak{A}}(m)\right)^{1 / q} \\
& \ll \sum_{k \geqslant \theta} 2^{-k r}\left(\sum_{\tau_{1}+\cdots+\tau_{s}=k} \sum_{m \in \rho(\tau)} \chi_{\mathfrak{A}}(m)\right)^{1 / q} .
\end{aligned}
$$


И, наконец, в силу леммы 11

$$
\Delta \ll R^{-1 / q} \sum_{k \geqslant \theta} 2^{-(r-1 / q) k} k^{(s-1) / q} \ll \frac{(\ln R)^{(s-1) / q}}{R^{r}} \ll \frac{(\ln T)^{s r+(s-1) / q}}{T^{r}}
$$

так что соотношение (2) доказано.

Перейдем к доказательству неравенства (3). Так как для всякого $x(t) \in H_{q, s}^{r}$ вьполнено (см. [16, с. 32])

$$
\left\|\sum_{m \in \rho(\tau)} \widehat{x}(m) e^{2 \pi i(m, t)}\right\|_{L^{q}(0,1)^{s}} \ll 2^{-\left(\tau_{1}+\cdots+\tau_{s}\right)}
$$

то, продолжая $(4)$, имеем $\left(q^{\prime}=q /(q-1)\right)$

$$
\begin{aligned}
\Delta & \ll \sum_{\tau_{1}+\cdots+\tau_{s} \geqslant \theta}\left(\sum_{m \in \rho(\tau)}|\widehat{x}(m)|^{q^{\prime}}\right)^{1 / q^{\prime}}\left(\sum_{m \in \rho(\tau)} \chi_{\mathfrak{A}}(m)\right)^{1 / q} \\
& \ll R^{-1 / q} \sum_{\tau_{1}+\cdots+\tau_{s} \geqslant \theta} 2^{\left(\tau_{1}+\cdots+\tau_{s}\right) 1 / q}\left\|\sum_{m \in \rho(\tau)} \widehat{x}(m) e^{2 \pi i(m, t)}\right\|_{L^{q}(0,1)^{s}} \\
& \ll R^{-1 / q} \sum_{\tau_{1}+\cdots+\tau_{s} \geqslant \theta} 2^{-(r-1 / q)\left(\tau_{1}+\cdots+\tau_{s}\right)} \\
& \ll R^{-r}(\ln R)^{s-1} \ll \frac{(\ln T)^{s r+(s-1)}}{T^{r}}
\end{aligned}
$$

т.е. (3) доказано. Теорема полностью доказана.

ЗАмЕчаниЕ 1. Сетка (1) полностью определяется заданием $(s+1)$-мерного целочисленного вектора $\left(p, a_{1}, \ldots, a_{s}\right)$, по которому легко выписьвается за $\asymp p$ элементарных арифметических операций, причем каждая координата каждого узла есть правильная дробь со знаменателем $p$.

ЗАмЕчАниЕ 2. Сетка (1), вьписываемая заこ раций, согласно алгоритму $1-5$ находится за $\leqslant c(s) p \ln \ln p$ элементарных арифметических операций.

ЗАМЕчАниЕ 3. Оценки (2) и (3) точны в степенной шкале (см. [1]-[5] и [7]).

ЗАмЕчАниЕ 4. Как это видно из доказательства теоремы, алгоритм 1-5 не зависит от принадлежности функций тому или иному классу. Другими словами, соответствующая этому алгоритму квадратурная формула автоматически реагирует на гладкость.

ЗАмЕчАниЕ 5. Все константы в данной работе являются эффективными. 


\section{СПИСОК ЦИТИРОВАННОЙ ЛИТЕРАТУРЫ}

[1] Фролов К. К. Оценки сверху погрешности квадратурных формул на классах функций 33 // Докл. АН СССР. 1976. Т. 231. № 4. С. 818-821.

[2] Быковский В. А. О правильном порядке погрешности оптимальных кубатурных формул в пространствах с доминирующей производной и квадратических отклонениях сеток. Препринт № 23. Владивосток: ВЦ ДВНЦ АН СССР, 1985.

[3] Темляков В.Н. Об одном приеме получения оценок снизу погрешностей квадратурных формул // Матем. сб. 1990. Т. 181. № 10. С. 1403-1413.

[4] Бахвалов Н. С. Оценки снизу асимптотических характеристик функций с доминирующей смешанной производной // Матем. заметки. 1972. Т. 12. №6. С. 655-664.

[5] Дубинин В. В. Об оптимальных формулах для классов функций с ограниченной смешанной производной // Матем. сб. 1992. Т. 183. № 7. С. 23-34.

[6] Коробов Н. М. О приближенном вычислении кратных интегралов // Докл. АН СССР. 1959. T. 124. №6. C. 1207-1210.

[7] Коробов Н. М. Теоретико-числовые методы в приближенном анализе. М.: Физматгиз, 1963.

[8] Hua Loo Keng, Wang Yuang. Applications of Number Theory of Numerical Analysis. Berlin-Heidelberg-N. Y.: Springer Verlag, 1981.

[9] Hlawka E., Firneis F., Zinterhof P. Zahlen-theoretische Methoden in der numerishen Mathematik. Wein-München-Oldenbourg, 1981.

[10] Кейперс Л., Нидеррейтер Г. Равномерное распределение. М.: Наука, 1985.

[11] Скриганов М. М. О решетках в полях алгебраических чисел // Докл. АН СССР. 1989. T. 306. №3. C. 353-355.

[12] Wang Yuang. Number theoretic method in numerical analysis // Contemp. Math. 1988. № 77. P. 63-82.

[13] Воронин С. М., Темиргалиев Н. О квадратурных формулах, связанных с дивизорами поля гауссовых чисел // Матем. заметки. 1989. Т. 46. № 2. С. 34-41.

[14] Темиргалиев Н. Применение теории дивизоров к численному интегрированию периодических функций многих переменных // Матем. сб. 1990. Т. 181. №4. С. 490-505.

[15] Эдвардс Р. Ряды Фурье в современном изложении. М.: Мир, 1985.

[16] Темляков В.Н. Приближение функций с ограниченной смешанной производной // Tp. МИАН. Т. 178. М.: Наука, 1986. 